S. N. Mukhopadhyay and S. Mitra*, Department of Mathematics, The University of Burdwan, Burdwan - 713104, India.

\title{
AN EXTENSION OF A THEOREM OF ASH ON GENERALIZED DIFFERENTIABILITY
}

\begin{abstract}
Let $A=\left\{b_{0}, b_{1}, \ldots, b_{k+\ell} ; a_{0}, a_{1}, \ldots, a_{k+\ell}\right\}$ be a system of $2(k+\ell+1)$ real numbers such that $b_{i} \neq b_{j}$ for $i \neq j$, satisfying $\sum_{i=0}^{k+\ell} a_{i} b_{i}^{p}=0$ for $p=0,1, \ldots, k-1$ and $\sum_{i=0}^{k+\ell} a_{i} b_{i}^{k}=L \neq 0$. It is proved that if $f$ is measurable, and if $\sum_{i=0}^{k+\ell} a_{i} f\left(x+b_{i} h\right)=O\left(|h|^{\lambda}\right)$ as $h \rightarrow 0$, where $\lambda>k-1$, at each point $x$ on a measurable set $E$ then the Peano derivative $f_{([\lambda])}$ exists finitely a.e. on $E$. This will extend a result of Ash [1]. It is further proved that if $p$ is a positive integer $\leq k-1$ and if the upper and lower approximate Peano derivatives of $f$ of order $p$ are finite on a set $E$ then $f_{(p)}$ exists a.e. on $E$.
\end{abstract}

\section{Introduction}

Throughout the paper $\mathbb{R}, \mathbb{N}$ and $\mathbb{N}^{+}$will denote the set of real numbers, the set of all non-negative integers, and the set of all positive integers respectively. The Lebesgue measure of a measurable set $E$ will be denoted by $\mu(E)$, and the Lebesgue outer measure of a set $H$ will be denoted by $\mu^{*}(H)$.

We shall consider $f: \mathbb{R} \rightarrow \mathbb{R}$. Recall that $f$ is said to have Peano derivative (resp. approximate Peano derivative) at $x$ of order $k$ if there exist real numbers $\alpha_{i}, 1 \leq i \leq k$, depending on $x$ and $f$ only, such that

$$
f(x+t)=f(x)+\sum_{i=1}^{k} \frac{t^{i} \alpha_{i}}{i !}+\frac{t^{k} \epsilon(x, t ; f)}{k !}
$$

Key Words: Peano derivative, approximate Peano derivative.

Mathematical Reviews subject classification: 26A24

Received by the editors January 29, 1998

* The work of the second author was supported by CSIR grant of INDIA. 
where

$$
\lim _{t \rightarrow 0} \epsilon(x, t ; f)=0 \quad\left(\text { resp. } \quad \operatorname{limap}_{t \rightarrow 0} \epsilon(x, t ; f)=0\right) .
$$

The number $\alpha_{k}$ is called the Peano derivative (resp. approximate Peano derivative) of $f$ at $x$ of order $k$ and is denoted by $f_{(k)}(x)$ (resp. $f_{(k), a}(x)$ ). For convenience, we take $\alpha_{0}=f(x)=f_{(0)}(x)=f_{(0), a}(x)$.

Suppose that $f$ has Peano derivative (resp. approximate Peano derivative) at $x$ of order $k$. For $t \neq 0$ write

$$
w_{k+1}(x, t ; f)=w_{k+1}(x, t)=(k+1) ! \frac{f(x+t)-\sum_{i=0}^{k} \frac{t^{i} \alpha_{i}}{i !}}{t^{k+1}} .
$$

The upper (resp. approximate upper) Peano derivative of $f$ at $x$ of order $k+1$ is defined by

$$
\bar{f}_{(k+1)}(x)=\limsup _{t \rightarrow 0} w_{k+1}(x, t)
$$

( respectively

$$
\left.\bar{f}_{(k+1), a}(x)=\limsup _{t \rightarrow 0} \operatorname{ap} w_{k+1}(x, t)\right) .
$$

The lower derivatives $\underline{f}_{(k+1)}(x)$ and $\underline{f}_{(k+1), a}(x)$ are defined analogously. If

$$
\bar{f}_{(k+1)}(x)=\underline{f}_{(k+1)}(x) \quad\left(\text { respectively } \bar{f}_{(k+1), a}(x)=\underline{f}_{(k+1), a}(x)\right)
$$

then the common value is called the Peano derivative (resp. approximate Peano derivative) of $f$ at $x$ (possibly infinite) of order $k+1$.

Definition 1.1. Let $k \in \mathbb{N}^{+}, \ell \in \mathbb{N}$ and $L \in \mathbb{R} \backslash\{0\}$. Let

$$
A=\left\{b_{0}, b_{1}, \ldots, b_{k+\ell} ; a_{0}, a_{1}, \ldots, a_{k+\ell}\right\}
$$

be a system of real numbers such that $b_{i} \neq b_{j}$ for $i \neq j, i, j=0,1, \ldots, k+\ell$, and

$$
\begin{aligned}
\sum_{i=0}^{k+\ell} a_{i} b_{i}^{p} & =0 \quad \text { for } \quad p=0,1, \ldots, k-1 \\
& =L \quad \text { for } \quad p=k
\end{aligned}
$$

For a fixed system $A$ in (1.1) satisfying (1.2), and for a function $f: \mathbb{R} \rightarrow \mathbb{R}$ we shall write

$$
\Phi_{k}(x, h)=\Phi_{k}(x, h ; f)=\Phi_{k}(x, h ; f ; A)=\sum_{i=0}^{k+\ell} a_{i} f\left(x+b_{i} h\right) .
$$


The generalized Riemann derivative of $f$ at $x$ of order $k$ with respect to the system $A$ is defined by

$$
\operatorname{GRD}_{k} f(x)=\operatorname{GRD}_{k} f(x, A)=\frac{k !}{L} \lim _{h \rightarrow 0} \frac{\Phi_{k}(x, h ; f ; A)}{h^{k}},
$$

if this limit exists. It can be shown that if the Peano derivative $f_{(k)}(x)$ exists finitely then $\mathrm{GRD}_{k} f(x, A)$ exists for every system $A$ in (1.1) satisfying $(1.2)$ and equals $f_{(k)}(x)$. The upper and lower derivatives $\overline{\operatorname{GRD}}_{k} f(x)$ and $\mathrm{GRD}_{k} f(x)$ are defined in the obvious way. Thus $A$ may be called the basis of a $k$ th order generalized derivative. The number $\ell$ is called its excess.

The following lemma is immediate.

Lemma 1.1. Let $\ell \in \mathbb{N}^{+}$and let there be $m(\leq \ell)$ zeros among the $a_{i}$ 's in (1.1). Let $A_{0}$ be obtained from $A$ by omitting those $a_{i}$ 's which are 0 and those $b_{i}$ 's which correspond to those $a_{i}$ 's. Then $A_{0}$ is a basis having excess only $l-m$ and

$$
\Phi_{k}(x, h ; f ; A)=\Phi_{k}\left(x, h ; f ; A_{0}\right)
$$

and therefore the $k$ th derivative with respect to $A$ is the same as the kth derivative with respect to $A_{0}$.

If $\ell=0$ and the $b_{i}$ 's are given then the $a_{i}$ 's are uniquely determined by (1.2). In fact $\left(b_{i}^{p}\right), 0 \leq i \leq k, 0 \leq p \leq k$, being a Van der Monde matrix, its determinant is given by

$$
\operatorname{det}\left(b_{i}^{p}\right)=\prod_{i<j}\left(b_{j}-b_{i}\right)
$$

and so if $\left(C_{r}^{k}\right)$ is the cofactor of $b_{r}^{k}$ in $\operatorname{det}\left(b_{i}^{p}\right)$ then $\left(C_{r}^{k}\right)$ is also Van der Monde and

$$
\operatorname{det}\left(C_{r}^{k}\right)=(-1)^{k+r} \prod_{i<j}^{\prime}\left(b_{j}-b_{i}\right)
$$

where $b_{r}$ never occurs in $\Pi^{\prime}$. Thus

$$
\frac{\operatorname{det}\left(b_{i}^{p}\right)}{\operatorname{det}\left(C_{r}^{k}\right)}=\prod_{i=0, i \neq r}^{k}\left(b_{r}-b_{i}\right)
$$

and

$$
a_{r}=L\left(\prod_{i=0, i \neq r}^{k}\left(b_{r}-b_{i}\right)\right)^{-1}, \quad 0 \leq r \leq k
$$


If in particular $L=k$ ! then the system (1.1) with (1.2) is considered by Ash [1] and it covers a wide class of $k$ th derivatives. The advantage of taking $L$ is that we can also accommodate the derivative $\tilde{D}_{k}$ considered in $[5, \mathrm{pp}$. 9-11]. Indeed, if

$$
L=2^{k-1} \prod_{i=1}^{k-1}\left(2^{k-1}-2^{i-1}\right) \quad \text { and } \quad \ell=0
$$

and if $b_{0}=0, b_{i}=2^{i-1}, 1 \leq i \leq k$, then the $k$ th derivative with respect to this system is the derivative $\tilde{D}_{k}$.

Now suppose that $\ell=0$. If $b_{i}=i+C$, where $C$ is a constant, then from (1.4) we have

$$
a_{i}=\frac{L}{k !}(-1)^{k-i}\left(\begin{array}{c}
k \\
i
\end{array}\right) .
$$

If on the other hand $\ell>0$ then the $b_{i}$ 's and the $k+1$ equations in (1.2) cannot determine the $a_{i}$ 's uniquely. It is clear that if $A$ is Riemann's symmetric system, i.e., $\ell=0, L=k !$ and $b_{i}=i-\frac{k}{2}$ (and so $a_{i}$ are as in (1.5)) then $\Phi_{k}$ becomes Riemann's symmetric difference of order $k$ given by

$$
\Delta_{k}(x, h ; f)=\sum_{i=0}^{k}(-1)^{k-i}\left(\begin{array}{c}
k \\
i
\end{array}\right) f(x+i h-k h / 2) .
$$

Marcinkiewicz and Zygmund proved in a deep theorem [5, Theorem 1] that if $f$ is measurable and

$$
\Delta_{k}(x, h ; f)=O\left(h^{k}\right), \quad \text { as } h \rightarrow 0
$$

for each $x$ on a measurable set $E$, then the Peano derivative $f_{(k)}$ exists finitely a.e. on $E$. We have extended in [6] the theorem of Marcinkiewicz and Zygmund cited above replacing $k$ at the right of (1.7) by any real number $\lambda>k-1$. More precisely, our theorem is:

Theorem 1.2. Let $k \in \mathbb{N}^{+}$and $\lambda \in \mathbb{R}$ be such that $\lambda>k-1$. Let $f$ be measurable. If

$$
\Delta_{k}(x, h ; f)=O\left(|h|^{\lambda}\right), \quad \text { as } h \rightarrow 0,
$$

for each point $x$ in a set $E \subset \mathbb{R}$, then $f_{([\lambda])}$ exists finitely a.e. on $E$, where $[\lambda]$ denotes the greatest integer not exceeding $\lambda$.

Ash [1] generalized the theorem of Marcinkiewich and Zygmund for any general system $A$ in (1.1) satisfying (1.2) with $L=k$ !. In the present paper we consider a general system $A$ in (1.1) satisfying (1.2), and consider the generalized difference $\Phi_{k}(x, h ; f ; A)$ instead of $\Delta_{k}(x, h ; f)$ and prove the analogue of Theorem 1.2. This will be an extension of Theorem 1 of [1]. 
Remark. In addition to [3] we wish to mention that there seems to be a difficulty in the assumption

$$
\left|\sum_{i=0}^{k+\ell} A_{i} f_{2}\left(x+a_{i} t\right)\right| \leq M|t|^{k} \quad \text { if }|t|<\delta \text { for all } x \in \Pi
$$

in $[1, \mathrm{p} .189]$. It may be noted that the similar assumption

$$
|\omega(x, t)|<M \quad \text { for } x \in \Pi,|t|<d
$$

in $[11$, Vol. II, p. 75$]$ can now be proved by taking

$$
G_{n}=\left\{x: x \in E_{k-1} ;\left|\omega_{k}(x, t)\right| \leq n \text { for } 0<|t|<\frac{1}{n}\right\}
$$

where $E_{k-1}$ is the set where $f_{(k-1)}$ exists, and noting the measurability of $G_{n}$ for all $n$ (cf. [7, p. 771]), and choosing $G_{m} \subset E, \mu\left(E \backslash G_{m}\right)<\frac{\epsilon}{2}$ and a perfect set $\Pi \subset G_{m}, \mu\left(G_{m} \backslash \Pi\right)<\frac{\epsilon}{2}$ and setting $M=m+1=\frac{1}{d}$. This approach will not work for (1.8) since the sets

$$
S_{n}=\left\{x:\left|\sum_{i=0}^{k+\ell} A_{i} f\left(x+a_{i} t\right)\right| \leq n|t|^{k} \quad \text { for } \quad|t|<\frac{1}{n}\right\}
$$

need not be measurable even for sufficiently large $n$. We show this in Example 1.5 which is an extension of Example 1 of [3]. We need a set $S$ of measure 0 such that $(S+S) / 2$ is non-measurable. For the proof of the existence of such a set the authors of [3] suggested a method and refered to a source not available to the readers. We give a proof in Theorem 1.4.

For any two sets $A, B,-A$ is the set of all $x$ such that $-x \in A$, and for a fixed $\tau \in \mathbb{R}, A+\tau$ is the set of all points $x+\tau$ such that $x \in A$, and $\tau A$ is the set of all $\tau x$ such that $x \in A$, and $A+B$ is the set of all points $x+y$ such that $x \in A$ and $y \in B$. For the definition of a Hamel basis we refer to $[10$, p. 411]. We need the following lemma which is a generalization of a result of Sierpinski [9] and is proved by Rubel [8]. We give a proof for completeness.

Lemma 1.3. There exists a bounded set $E$ of Lebesgue measure 0 , but $E+E$ is non-measurable.

Proof. Let $C$ be the Cantor ternary set in $[0,1]$. Let $r \in[0,1]$ and let $0 . a_{1} a_{2} \ldots$, where $a_{i}=0,1$ or 2 , be the ternary expansion of $r / 2$. Define $c_{i}$ and $c_{i}^{\prime}$ for each $i$ such that $\left(c_{i}, c_{i}^{\prime}\right)=(0,0)$ if $a_{i}=0,\left(c_{i}, c_{i}^{\prime}\right)=(2,0)$ if $a_{i}=1$, and 
$\left(c_{i}, c_{i}^{\prime}\right)=(2,2)$ if $a_{i}=2$. Then $c=0 . c_{1} c_{2} \ldots$ and $c^{\prime}=0 . c_{1}^{\prime} c_{2}^{\prime} \ldots$ are points of $C$ and

$$
\frac{r}{2}=\frac{c+c^{\prime}}{2} \quad \text { giving } \quad r=c+c^{\prime} .
$$

Thus $[0,1] \subset C+C$. Hence $C \pm C \pm C \pm \ldots=\mathbb{R}$ and therefore $C$ contains a Hamel basis $H$. Let

$$
E_{0}=H \cup(-H) \cup\{0\} ; E_{n+1}=E_{n}+E_{n} \text { for } n=0,1,2, \ldots .
$$

Then

$$
\mathbb{R}=\bigcup_{n=0}^{\infty} \bigcup_{m=1}^{\infty} \frac{1}{m} E_{n}
$$

For, if $r \in \mathbb{R}$, then there are $h_{1}, h_{2}, \ldots, h_{p} \in H$ and rationals $\rho_{1}, \rho_{2}, \ldots, \rho_{p}$ such that

$$
r=\sum_{i=1}^{p} \rho_{i} h_{i}=\frac{1}{d} \sum_{i=1}^{p} e_{i} h_{i},
$$

where $\rho_{i}=\frac{e_{i}}{d}$ with $\left|e_{i}\right| \in \mathbb{N}^{+}, d \in \mathbb{N}^{+}$, and so

$$
r \in \frac{1}{d} E_{n} \quad \text { if } \quad 2^{n} \geq \sum_{i=1}^{p}\left|e_{i}\right|
$$

Hence all sets $E_{n}$ cannot be of measure 0 . Let $n_{0}$ be the smallest of $n$ for which $E_{n}$ has positive outer measure. Since $E_{0}$ is of measure $0, n_{0} \geq 1$. If possible, let $E_{n_{0}}$ be measurable. Since $E_{n_{0}}=-E_{n_{0}}, E_{n_{0}+1}=E_{n_{0}}-E_{n_{0}}$. So by [4, p. 68], $E_{n_{0}+1}$ contains an open interval $I$ containing the origin. Let $h \in H$. Then we can find an integer $j \geq 2$ such that $\frac{h}{j} \in I$ and hence $\frac{h}{j} \in E_{n_{0}+1}=E_{n_{0}}+E_{n_{0}}$. Since every element of $E_{n_{0}}$ is a linear combination of elements of $E_{o}$ and hence of $H$ with integral coefficients, $\frac{h}{j}$ is a linear combination of elements of $H$ with integral coefficients. But this is a contradiction since $H$, being a Hamel basis, is a linearly independent set with rational coefficients. Therefore, $E_{n_{0}}$ is not measurable. Putting $E=E_{n_{0}-1}$ the proof is complete.

Theorem 1.4. For any bounded interval $I$ there is a set $S \subset I$ of measure 0 , but $\frac{S+S}{2}$ is non-measurable.

Proof. Let $I=[a, b], \alpha=\inf E, \beta=\sup E$, where $E$ is the set of Lemma 1.3. Let

$$
S=\left\{\frac{(b-a)(x-\alpha)}{\beta-\alpha}+a: x \in E\right\} .
$$

Then $S$ satisfies the requirements. 
Example 1.5. There exists a measurable function $f: \mathbb{R} \rightarrow \mathbb{R}$ such that for each $n \in \mathbb{N}^{+}$the set

$$
E_{n}=\left\{x: \frac{|f(x+h)-2 f(x)+f(x-h)|}{h^{2}} \leq n \text { for } 0<|h|<\frac{1}{n}\right\}
$$

is non-measurable.

Proof. Let $n \in \mathbb{N}^{+}$and let

$$
a_{n}=\frac{1}{n}-\frac{1}{8 n^{2}}, \quad \delta_{n}=\frac{1}{32 n^{2}} .
$$

Let $I_{n}$ be the closed interval with center $2 n-1$ and length $2 \delta_{n}$. By Theorem 1.4 there is a set $S_{n} \subset I_{n}$ of measure 0 such that $\frac{S_{n}+S_{n}}{2}$ is non-measurable. Let

$$
f_{n}=\frac{1}{2 n} \chi_{\left(S_{n}-a_{n}\right) \cup\left(S_{n}+a_{n}\right)},
$$

where $\chi_{E}$ is the characteristic function of $E$. Since $S_{n}$ is of measure $0, f_{n}$ is measurable. Applying similar arguments as in [3] with $a, \delta, S$ and $\left[\frac{1}{2}-\delta, \frac{1}{2}+\delta\right]$ being replaced by $a_{n}, \delta_{n}, S_{n}$ and $I_{n}$ respectively, it can be shown that

$$
\begin{aligned}
\frac{S_{n}+S_{n}}{2}=\left\{x: x \in I_{n} ; \frac{\left|f_{n}(x+h)-2 f_{n}(x)+f_{n}(x-h)\right|}{h^{2}}\right. & >n, \\
& \text { for some } \left.h, 0<|h|<\frac{1}{n}\right\} .
\end{aligned}
$$

Hence the set

$$
\left\{x: x \in I_{n} ; \quad \frac{\left|f_{n}(x+h)-2 f_{n}(x)+f_{n}(x-h)\right|}{h^{2}} \leq n, \text { for } 0<|h|<\frac{1}{n}\right\}
$$

is non-measurable. Let $f=\sum_{n=1}^{\infty} f_{n}$. Then for each $\nu \in \mathbb{N}^{+}$the set

$$
E_{\nu}=\left\{x: \frac{|f(x+h)-2 f(x)+f(x-h)|}{h^{2}} \leq \nu, \text { for } 0<|h|<\frac{1}{\nu}\right\}
$$

is non-measurable. For, if possible, suppose $E_{\nu}$ is measurable. Then $E_{\nu} \cap I_{\nu}$ is measurable. But

$$
\begin{gathered}
E_{\nu} \cap I_{\nu}= \\
=\left\{x: x \in I_{\nu} ; \quad \frac{\left|f_{\nu}(x+h)-2 f_{\nu}(x)+f_{\nu}(x-h)\right|}{h^{2}} \leq \nu, \text { for } 0<|h|<\frac{1}{\nu}\right\}
\end{gathered}
$$

which is non-measurable, giving a contradiction.

We shall follow the approach of Ash [1] with essential modifications. 


\section{Auxiliary Results}

We need the following results from [3]:

Lemma 2.1. Let 0 be a point of outer density of $E$, let $\alpha, \beta \in \mathbb{R}$ with $\beta \neq 0$ and let $\epsilon>0$. For each $u>0$ set

$$
B_{u}=\{v \in[u, 2 u]: \alpha u+\beta v \in E\} .
$$

Then there is a $\delta>0$ such that if $0<u<\delta$, then $\mu^{*}\left(B_{u}\right)>u(1-\epsilon)$.

Theorem 2.2. Let $f$ be measurable and let $n \in \mathbb{N}^{+}$. Suppose that $\alpha_{i}, \beta_{i}$, $i=0,1, \ldots, n$ are real numbers such that $\beta_{i} \neq \beta_{j}$ for $i \neq j$ and for some $i \in\{0,1, \ldots, n\}, \alpha_{i} \neq 0, \beta_{i} \neq 0$. If

$$
\sum_{i=0}^{n} \alpha_{i} f\left(x+\beta_{i} t\right)=O(1), \quad \text { as } t \rightarrow 0
$$

for $x \in E \subset \mathbb{R}$, then $f$ is bounded in a neighborhood of almost every point $x \in E$.

Theorem 2.3. Let the hypotheses of Theorem 2.2 hold. If $\alpha \geq 0$ and

$$
\sum_{i=0}^{n} \alpha_{i} f\left(x+\beta_{i} t\right)=O\left(|t|^{\alpha}\right), \quad \text { as } t \rightarrow 0
$$

for all $x \in E \subset \mathbb{R}$, then for each $\beta \in \mathbb{R}$

$$
\sum_{i=0}^{n} \alpha_{i} f\left(x+\left(\beta_{i}-\beta\right) t\right)=O\left(|t|^{\alpha}\right), \quad \text { as } t \rightarrow 0
$$

for almost every $x \in E$.

The theorem is true if " $O$ " is replaced by " $O$ ".

The above results are respectively Lemma 1, Theorem 2 and Theorem 3 of [3].

Lemma 2.4. Let $f$ be measurable and let the Peano derivative $f_{(k-1)}(x)$ of $f$ at $x$ of order $k-1$ exist for each $x$ in a set $E \subset \mathbb{R}$. If

$$
f(x+h)-\sum_{i=0}^{k-1} \frac{h^{i} f_{(i)}(x)}{i !}=O\left(h^{k}\right), \quad \text { as } h \rightarrow 0
$$

for $x \in E$ then $f_{(k)}$ exists a.e. on $E$. 
Proof. The proof is in [5, Lemma 7] and discussed in [6, Theorem MZ1] when $E$ is measurable. When $E$ is non-measurable, let

$E_{1}=\left\{x: f_{(k-1)}(x)\right.$ exists and $f(x+h)-\sum_{i=0}^{k-1} \frac{h^{i} f_{(i)}(x)}{i !}=O\left(h^{k}\right)$ as $\left.h \rightarrow 0\right\}$.

Then since the upper and lower Peano derivatives are measurable, $E_{1}$ is measurable and so $f_{(k)}$ exists a.e. on $E_{1}$. Since $E \subset E_{1}$, the result follows.

\section{Main Results}

The $C_{r} P$-integral, which is introduced by J. C. Burkill and used in the following lemma, can be found in [2]. Indeed, any integral will suffice if integrability of $f$ implies measurability of $f$.

Lemma 3.1. Let $f$ be $C_{r} P$-integrable in every finite interval on $\mathbb{R}$ for some $r \in \mathbb{N}^{+}$. Let

$$
\Phi_{k}(x, h ; f ; A)=\sum_{i=0}^{k+\ell} a_{i} f\left(x+b_{i} h\right)=O\left(|h|^{\lambda}\right), \quad \text { as } h \rightarrow 0,
$$

where $\lambda \geq 0$ at each point $x$ on a set $E \subset \mathbb{R}$. Then there is $s \in \mathbb{N}$ such that

$$
\Delta_{k+s}\left(x, h ; F_{s}\right)=O\left(|h|^{\lambda+s}\right), \text { as } h \rightarrow 0,
$$

for almost all $x \in E$ where $F_{s}$ is the sth indefinite $C_{r} P$-integral of $f$, i.e.,

$$
\begin{aligned}
& F_{0}(x)=f(x) ; \quad F_{1}(x)=\int_{0}^{x} f(t) d t ; \\
& F_{s}(x)=\frac{1}{(s-1) !} \int_{0}^{x}(x-t)^{s-1} f(t) d t, \quad \text { for } s \geq 2 .
\end{aligned}
$$

Proof. We note that, since $f$ is $C_{r} P$-integrable, it is measurable [2, Proposition 4.7]. We may suppose that $b_{0}<b_{1}<\ldots<b_{k+\ell}$. By Theorem 2.3 we may further suppose that $b_{0}=1$. We consider the following cases:

CASE I. Let $\ell=0, b_{i} \in \mathbb{N}^{+}$for $i=1,2, \ldots, k$. Then $b_{k}=s+k+1$ for some $s \in \mathbb{N}$. If $s=0$ then $b_{i}=i+1$ for $i=0,1, \ldots, k$, and so the $a_{i}$ 's are given by (1.5). Hence from (3.1) and Theorem 2.3 (with $\alpha_{i}=a_{i}, \beta_{i}=i+1$, $\left.\beta=\frac{k}{2}+1, \alpha=\lambda\right)$, we get (3.2) for $s=0$.

If $s>0$ there are $s$ gaps in $b_{0}, b_{1}, \ldots, b_{k}$. Let $n_{1}$ be the smallest positive integer in $\left(b_{0}, b_{k}\right)$ such that $n_{1} \notin\left\{b_{0}, b_{1}, \ldots, b_{k}\right\}$. Applying Theorem 2.3 in 
(3.1) with $\alpha_{i}=a_{i}, \beta_{i}=b_{i}, \beta=n_{1}$ and $\alpha=\lambda$, we have

$$
\sum_{i=0}^{k} a_{i} f\left(x+\left(b_{i}-n_{1}\right) h\right)=O\left(|h|^{\lambda}\right), \quad \text { as } h \rightarrow 0,
$$

for almost all $x \in E$, and integrating with respect to $h$ from 0 to $t,|t|$ being sufficiently small, we have

$$
\begin{gathered}
\sum_{i=0}^{k} \frac{a_{i}}{b_{i}-n_{1}} F_{1}\left(x+\left(b_{i}-n_{1}\right) t\right)-\left(\sum_{i=0}^{k} \frac{a_{i}}{b_{i}-n_{1}}\right) F_{1}(x)= \\
=O\left(|t|^{\lambda+1}\right), \quad \text { as } t \rightarrow 0,
\end{gathered}
$$

for almost all $x \in E$. By (1.2) all the $a_{i}$ 's cannot be 0 , and so applying Theorem 2.3 in (3.4) with $\beta=-n_{1}, \alpha=\lambda+1$ and $\alpha_{i}=\frac{a_{i}}{b_{i}-n_{1}}, \beta_{i}=b_{i}-n_{1}$ for $i=0,1, \ldots, k$ and $\alpha_{k+1}=-\sum_{i=0}^{k} \frac{a_{i}}{b_{i}-n_{1}}, \beta_{k+1}=0$, we have

$$
\begin{gathered}
\sum_{i=0}^{k} \frac{a_{i}}{b_{i}-n_{1}} F_{1}\left(x+b_{i} t\right)-\left(\sum_{i=0}^{k} \frac{a_{i}}{b_{i}-n_{1}}\right) F_{1}\left(x+n_{1} t\right)= \\
=O\left(|t|^{\lambda+1}\right), \quad \text { as } t \rightarrow 0,
\end{gathered}
$$

for almost all $x \in E$. It is easy to check that the system

$$
A_{1}=\left\{b_{0}, b_{1}, \ldots, b_{k}, n_{1}, \frac{a_{0}}{b_{0}-n_{1}} ; \frac{a_{1}}{b_{1}-n_{1}}, \ldots, \frac{a_{k}}{b_{k}-n_{1}},-\sum_{i=0}^{k} \frac{a_{i}}{b_{i}-n_{1}}\right\}
$$

satisfies the condition (1.2) with $k$ replaced by $k+1$. Hence from (3.5) we observe that

$$
\Phi_{k+1}\left(x, h ; F_{1} ; A_{1}\right)=O\left(|h|^{\lambda+1}\right), \quad \text { as } h \rightarrow 0,
$$

for almost all $x \in E$. The numbers $b_{0}, b_{1}, \ldots, b_{k}, n_{1}$ have one fewer gap than $b_{0}, b_{1}, \ldots, b_{k}$ and also the excess is still 0 . So, if $s=1$, the proof is completed as in the first paragraph replacing $k$ by $k+1$ and $A$ by $A_{1}$. Otherwise, choose the smallest positive integer $n_{2}$ in $\left(b_{0}, b_{k}\right)$ such that $n_{2} \notin$ $\left\{b_{o}, b_{1}, \ldots, b_{k}, n_{1}\right\}$, and repeating this process $s-1$ more times, we obtain the numbers $b_{0}, b_{1}, \ldots, b_{k}, n_{1}, \ldots, n_{s}$ which have no gap, and we obtain the system $A_{s}$ such that

$$
\Phi_{k+s}\left(x, h ; F_{s} ; A_{s}\right)=O\left(|h|^{\lambda+s}\right), \quad \text { as } h \rightarrow 0,
$$

for almost all $x \in E$. The proof is completed as in the first paragraph. 
CASE II. Let $\ell \in \mathbb{N}^{+}, b_{i} \in \mathbb{N}^{+}$for $i=1,2, \ldots, k+\ell$. By employing the process of filling in the gaps employed in Case I, we may suppose that $b_{i}=i+1, i=1,2, \ldots, k+\ell$. It may be noted that the process of filling never increases the excess. Hence (3.1) reduces to

$$
\sum_{i=1}^{r} a_{i-1} f(x+i h)=O\left(|h|^{\lambda}\right), \quad \text { as } h \rightarrow 0 \text { for } x \in E,
$$

where $r=k+\ell+1$. Applying Theorem 2.3 in (3.6) with $\beta=r+1, \alpha=\lambda$, we have

$$
\sum_{i=1}^{r} a_{i-1} f(x+(i-(r+1)) h)=O\left(|h|^{\lambda}\right), \quad \text { as } h \rightarrow 0,
$$

for almost all $x \in E$. Integrating (3.6) and (3.7) with respect to $h$ from 0 to $t$, we have

$$
\begin{gathered}
\sum_{i=1}^{r} \frac{a_{i-1}}{i} F_{1}(x+i t)-\left(\sum_{i=1}^{r} \frac{a_{i-1}}{i}\right) F_{1}(x)= \\
=O\left(|t|^{\lambda+1}\right), \quad \text { as } t \rightarrow 0,
\end{gathered}
$$

for almost all $x \in E$, and

$$
\begin{gathered}
\sum_{j=1}^{r} \frac{a_{j-1}}{j-r-1} F_{1}(x+(j-r-1) t)-\left(\sum_{j=1}^{r} \frac{a_{j-1}}{j-r-1}\right) F_{1}(x)= \\
=O\left(|t|^{\lambda+1}\right), \quad \text { as } t \rightarrow 0,
\end{gathered}
$$

for almost all $x \in E$. Applying Theorem 2.3 to (3.9) with $\beta=-r, \alpha=\lambda+1$ and changing indices by setting $i=j-1$, we have

$$
\begin{gathered}
\sum_{i=0}^{r-1} \frac{a_{i}}{i-r} F_{1}(x+i t)-\left(\sum_{i=0}^{r-1} \frac{a_{i}}{i-r}\right) F_{1}(x+r t)= \\
=O\left(|t|^{\lambda+1}\right), \quad \text { as } t \rightarrow 0,
\end{gathered}
$$

for almost all $x \in E$. If possible, suppose that the coefficients of $F_{1}(x+i t)$, $0 \leq i \leq r$, in (3.8) and (3.10) are proportional. Then there is $\rho \in \mathbb{R} \backslash\{0\}$ such that

$$
\begin{aligned}
-\sum_{i=1}^{r} \frac{a_{i-1}}{i} & =-\frac{\rho a_{0}}{r} ; \\
\frac{a_{i-1}}{i} & =\frac{\rho a_{i}}{i-r}, \quad 1 \leq i \leq r-1 ; \\
\frac{a_{r-1}}{r} & =-\rho \sum_{i=0}^{r-1} \frac{a_{i}}{i-r} .
\end{aligned}
$$


It can be verified that the following two systems

$$
\begin{aligned}
& B_{1}=\left\{0,1, \ldots, r ;-\sum_{i=1}^{r} \frac{a_{i-1}}{i}, a_{0}, \frac{a_{1}}{2}, \ldots, \frac{a_{r-1}}{r}\right\} \\
& B_{2}=\left\{0,1, \ldots, r ; \frac{a_{0}}{-r}, \frac{a_{1}}{1-r}, \ldots, \frac{a_{r-1}}{-1},-\sum_{i=0}^{r-1} \frac{a_{i}}{i-r}\right\},
\end{aligned}
$$

which correspond to (3.8) and (3.10) respectively, satisfy the conditions (1.2) with $k$ replaced by $k+1$. In fact, it is easy for $B_{1}$. For $B_{2}$ note that

$$
\begin{gathered}
\sum_{i=0}^{r-1} \frac{a_{i}}{i-r} i^{p}-\left(\sum_{i=0}^{r-1} \frac{a_{i}}{i-r}\right) r^{p}= \\
=\sum_{i=0}^{r-1} a_{i} \sum_{\nu=0}^{p-1} i^{p-1-\nu} r^{\nu}=\sum_{\nu=0}^{p-1} r^{\nu} \sum_{j=1}^{r} a_{j-1}(j-1)^{p-1-\nu} \\
=\sum_{\nu=0}^{p-1} r^{\nu} \sum_{\mu=0}^{p-1-\nu}(-1)^{p-1-\nu-\mu}\left(\begin{array}{c}
p-1-\nu \\
\mu
\end{array}\right) \sum_{j=1}^{r} a_{j-1} j^{\mu},
\end{gathered}
$$

and since the last sum is 0 for $\mu=0,1, \ldots, k-1$ and $L$ for $\mu=k$, it is 0 if $p=0,1, \ldots, k$, and it is $L$ if $p=k+1$, proving the assertion. Hence from (3.11), (3.12) and the last condition of (1.2), we have $\rho L=L$ showing that $\rho=1$. Hence from (3.11)

$$
a_{i}=-\frac{r-i}{i} a_{i-1} \text { for } 1 \leq i \leq r-1,
$$

and hence

$$
\begin{aligned}
\sum_{i=0}^{r-1} a_{i}(i+1)^{k} & =a_{0} \sum_{i=0}^{r-1}(-1)^{i}\left(\begin{array}{c}
r-1 \\
i
\end{array}\right)(i+1)^{k} \\
& =a_{0} \sum_{i=0}^{r-1}(-1)^{i}\left(\begin{array}{c}
r-1 \\
i
\end{array}\right) \sum_{\nu=0}^{k}\left(\begin{array}{c}
k \\
\nu
\end{array}\right) i^{\nu} \\
& =a_{0} \sum_{\nu=0}^{k}\left(\begin{array}{c}
k \\
\nu
\end{array}\right) \sum_{i=0}^{r-1}(-1)^{i}\left(\begin{array}{c}
r-1 \\
i
\end{array}\right) i^{\nu} \\
& =(-1)^{r-1} a_{0} \sum_{\nu=0}^{k}\left(\begin{array}{c}
k \\
\nu
\end{array}\right) \sum_{i=0}^{r-1}(-1)^{r-1-i}\left(\begin{array}{c}
r-1 \\
i
\end{array}\right) i^{\nu}
\end{aligned}
$$


Since

$$
\begin{aligned}
\sum_{i=0}^{m}(-1)^{m-i}\left(\begin{array}{c}
m \\
i
\end{array}\right) i^{p} & =0 \quad \text { for } p=0,1, \ldots, m-1 \\
& =m ! \text { for } p=m
\end{aligned}
$$

(cf. (1.5) and (1.2)), and since $r-1=k+\ell>k$, the inner summation at the right of $(3.13)$ is 0 for $\nu=0,1, \ldots, k$, and so the right side of (3.13) is 0 . But since $b_{i}=i+1$ and the system $\left\{b_{0}, b_{1}, \ldots, b_{k+\ell} ; a_{0}, a_{1}, \ldots, a_{k+\ell}\right\}$ satisfies (1.2), the left hand side of (3.13) is $L \neq 0$, which is a contradiction. Therefore the coefficients of $F_{1}(x+i t), 0 \leq i \leq r$, in (3.8) and (3.10) are not proportional. Therefore denoting the coefficients of $F(x+i t), 0 \leq i \leq r$, in (3.8) and (3.10) by $p_{i}$ and $q_{i}$ respectively, we conclude that there is an $i_{0} \in\{0,1, \ldots, r\}$ such that $p_{i_{0}}$ and $q_{i_{0}}$ are not equal. Set

$$
\gamma=q_{i_{0}}\left(q_{i_{0}}-p_{i_{0}}\right)^{-1}, \quad \delta=-p_{i_{0}}\left(q_{i_{0}}-p_{i_{0}}\right)^{-1}
$$

then $\gamma+\delta=1$ and $p_{i_{0}} \gamma+q_{i_{0}} \delta=0$. Since (3.8) and (3.10) can be written as

$$
\Phi_{k+1}\left(x, h ; F_{1} ; B_{i}\right)=O\left(|t|^{\lambda+1}\right), \quad \text { as } t \rightarrow 0, \quad i=1,2,
$$

for almost all $x \in E$, where $B_{1}$ and $B_{2}$ are given in (3.12), we have

$$
\gamma \Phi_{k+1}\left(x, h ; F_{1} ; B_{1}\right)+\delta \Phi_{k+1}\left(x, h ; F_{1} ; B_{2}\right)=O\left(|t|^{\lambda+1}\right) \text { as } t \rightarrow 0
$$

for almost all $x \in E$. Let $B$ be obtained by adding $\gamma$ times the elements of $B_{1}$ with $\delta$ times the corresponding elements of $B_{2}$. Since $\gamma+\delta=1$, the first group of $r+1$ elements of $B$ are $0,1, \ldots, r$, and the second group of $r+1$ elements of $B$ are $\gamma p_{i}+\delta q_{i}, 0 \leq i \leq r$. Let $B_{0}$ be obtained by omitting from $B$ those $\gamma p_{i}+\delta q_{i}$ 's for which $\gamma p_{i}+\delta q_{i}=0$ and the corresponding $i$ 's. Then by Lemma 1.1 and by (3.14)

$$
\Phi_{k+1}\left(x, h ; F_{1} ; B_{0}\right)=\Phi_{k+1}\left(x, h ; F_{1} ; B\right)=O\left(|t|^{\lambda+1}\right) \text { as } t \rightarrow 0,
$$

for almost all $x \in E$, and therefore, since $\gamma p_{i_{0}}+\delta q_{i_{0}}=0, B_{0}$ has excess $\leq \ell-1$. Repeating this process at most $\ell-1$ more times, this case reduces to Case $\mathrm{I}$.

CASE III. Let $\ell \in \mathbb{N}^{+}$and $b_{i}$ 's be arbitrary reals for $1 \leq i \leq k+\ell$. (Note that we have assumed that $b_{0}=1<b_{1}<\ldots<b_{k+\ell}$.) Let $b_{i_{0}}$ be the smallest of $b_{1}, b_{2}, \ldots, b_{k+\ell}$ which is not an integer. Let $n_{1}, n_{2} \in \mathbb{N}^{+} \backslash\left\{b_{0}, b_{1}, \ldots, b_{k+\ell}\right\}$, $n_{1} \neq n_{2}$. Applying Theorem 2.3 with $\beta=n_{j}, j=1,2, \alpha=\lambda$ in (3.1), we have

$$
\sum_{i=0}^{k+\ell} a_{i} f\left(x+\left(b_{i}-n_{j}\right) h\right)=O\left(|h|^{\lambda}\right), \quad \text { as } h \rightarrow 0, \quad j=1,2,
$$


for almost all $x \in E$. Integrating (3.15.j) with respect to $h$ from 0 to $t$, and then applying Theorem 2.3 with $\beta=-n_{j}, j=1,2, \alpha=\lambda+1$, we have

$$
\begin{gathered}
\sum_{i=0}^{k+\ell} \frac{a_{i}}{b_{i}-n_{j}} F_{1}\left(x+b_{i} t\right)-\left(\sum_{i=0}^{k+\ell} \frac{a_{i}}{b_{i}-n_{j}}\right) F_{1}\left(x+n_{j} t\right)= \\
=O\left(|t|^{\lambda+1}\right), \quad \text { as } t \rightarrow 0, j=1,2,
\end{gathered}
$$

for almost all $x \in E$. Set

$$
p=\frac{b_{i_{0}}-n_{1}}{n_{2}-n_{1}} \quad \text { and } \quad q=\frac{n_{2}-b_{i_{0}}}{n_{2}-n_{1}} .
$$

Then

$$
p+q=1 \quad \text { and } \quad p \frac{a_{i_{0}}}{b_{i_{0}}-n_{1}}+q \frac{a_{i_{0}}}{b_{i_{0}}-n_{2}}=0 .
$$

Adding (3.16.1) multiplied by $p$ with (3.16.2) multiplied by $q$ we have

$$
\Phi_{k+1}\left(x, h ; F_{1} ; C_{1}\right)=O\left(|h|^{\lambda+1}\right), \quad \text { as } h \rightarrow 0,
$$

for almost all $x \in E$, where

$$
\begin{aligned}
C_{1}= & \left\{b_{0}, \ldots, b_{i_{0}}-1, b_{i_{0}+1}, \ldots, b_{k+\ell}, n_{1}, n_{2} ; p \frac{a_{0}}{b_{0}-n_{1}}+q \frac{a_{0}}{b_{0}-n_{2}},\right. \\
& \ldots, p \frac{a_{i_{0}-1}}{b_{i_{0}-1}-n_{1}}+q \frac{a_{i_{0}-1}}{b_{i_{0}-1}-n_{2}}, p \frac{a_{i_{0}+1}}{b_{i_{0}+1}-n_{1}}+q \frac{a_{i_{0}+1}}{b_{i_{0}+1}-n_{2}}, \\
& \left.\ldots, p \frac{a_{k+\ell}}{b_{k+\ell}-n_{1}}+q \frac{a_{k+\ell}}{b_{k+\ell}-n_{2}},-p \sum_{i=0}^{k+\ell} \frac{a_{i}}{b_{i}-n_{1}},-q \sum_{i=0}^{k+\ell} \frac{a_{i}}{b_{i}-n_{2}}\right\} .
\end{aligned}
$$

The system $C_{1}$ satisfies (1.2) with $k$ replaced by $k+1$. Indeed, using (3.17) we have

$$
\sum_{\substack{i=0 \\ i \neq i_{0}}}^{k+\ell}\left(p \frac{a_{i}}{b_{i}-n_{1}}+q \frac{a_{i}}{b_{i}-n_{2}}\right) b_{i}^{s}-\sum_{i=0}^{k+\ell} p \frac{a_{i}}{b_{i}-n_{1}} n_{1}^{s}-\sum_{i=0}^{k+\ell} q \frac{a_{i}}{b_{i}-n_{2}} n_{2}^{s}
$$

which is 0 if $s=0$, and if $1 \leq s \leq k+1$, then this is

$$
\begin{aligned}
& =\sum_{i=0}^{k+\ell}\left(p \frac{a_{i}\left(b_{i}^{s}-n_{1}^{s}\right)}{b_{i}-n_{1}}+q \frac{a_{i}\left(b_{i}^{s}-n_{2}^{s}\right)}{b_{i}-n_{2}}\right) \\
& =\sum_{i=0}^{k+\ell}\left(p a_{i} \sum_{j=0}^{s-1} b_{i}^{s-1-j} n_{1}^{j}+q a_{i} \sum_{j=0}^{s-1} b_{i}^{s-1-j} n_{2}^{j}\right) \\
& =\sum_{j=0}^{s-1}\left(p n_{1}^{j}+q n_{2}^{j}\right) \sum_{i=0}^{k+\ell} a_{i} b_{i}^{s-1-j}=\sum_{i=0}^{k+\ell} a_{i} b_{i}^{s-1},
\end{aligned}
$$


which is 0 if $1 \leq s<k+1$ and is $L$ if $s=k+1$. So, we have removed the non-integer $b_{i_{0}}$ and got $C_{1}$. We next pick the smallest of $b_{i_{0}+1}, \ldots, b_{k+\ell}$, say $b_{i_{1}}$, which is not an integer, and choose $n_{3}, n_{4} \in \mathbb{N}^{+} \backslash\left\{b_{0}, b_{1}, \ldots, b_{k+\ell}, n_{1}, n_{2}\right\}$, $n_{3} \neq n_{4}$, and repeat the above argument to get a system $C_{2}$ which contains $n_{1}, n_{2}, n_{3}, n_{4}$, instead of $b_{i_{0}}, b_{i_{1}}$ of $A$ such that

$$
\Phi_{k+2}\left(x, h ; F_{2} ; C_{2}\right)=O\left(|h|^{\lambda+2}\right), \quad \text { as } h \rightarrow 0,
$$

for almost all $x \in E$. After repeating the process we get a system $C_{u}$, where $1 \leq u \leq k+\ell$, of $2(1+k+\ell+u)$ elements in which the first set of $1+k+\ell+u$ elements are all in $\mathbb{N}^{+}$, and for which

$$
\Phi_{k+u}\left(x, h ; F_{u} ; C_{u}\right)=O\left(|h|^{\lambda+u}\right), \quad \text { as } h \rightarrow 0,
$$

for almost all $x \in E$. After rearranging the elements of $C_{u}$, this case now reduces to Case II. This completes the proof.

Theorem 3.2. Let $f$ be measurable. If

$$
\Phi_{k}(x, h ; f ; A)=O\left(|h|^{\lambda}\right), \quad \text { as } h \rightarrow 0,
$$

where $\lambda>k-1$, at each point $x$ in a measurable set $E \subset \mathbb{R}$, then $f_{([\lambda])}$ exists finitely a.e. on $E,[\lambda]$ being the greatest integer not exceeding $\lambda$.

Proof. We may suppose that $E$ is bounded. By Theorem 2.2 there is a measurable set $E_{1} \subset E$ such that $\mu\left(E_{1}\right)=\mu(E)$, and for each $x \in E_{1}$ there exist $\delta(x)>0$ and $M(x)$ with

$$
|f(t)| \leq M(x) \quad \text { for } \quad t \in(x-\delta(x), x+\delta(x)) .
$$

Let $\epsilon_{1}, \epsilon_{2}$ be arbitrary positive numbers. Then there is a closed set $E_{2} \subset E_{1}$ such that $\mu\left(E_{1} \backslash E_{2}\right)<\epsilon_{1}$, and so by the compactness of $E_{2}$ there exist open intervals $I_{1}, I_{2}, \ldots, I_{n}$ such that $E_{2} \subset \cup_{i=1}^{n} I_{i}$ and $f$ is bounded on $\cup_{i=1}^{n} I_{i}$. Clearly $f$ is bounded on the closure $\bar{I}=\bar{\cup}_{i} I_{i}$. Let $\psi=f$ on $\bar{I}$ and $=0$ outside $\bar{I}$. Then $\psi$ is Lebesgue integrable and a fortiori $C_{r} P$-integrable on every finite interval in $\mathbb{R}$. Then by Lemma 3.1, there exist $s \in \mathbb{N}$ and a set $E_{3} \subset E_{2}$ such that $\mu\left(E_{3}\right)=\mu\left(E_{2}\right)$ and

$$
\Delta_{k+s}\left(x, t ; \psi_{s}\right)=O\left(|t|^{\lambda+s}\right), \quad \text { as } t \rightarrow 0,
$$

for all $x \in E_{3}$, where $\psi_{s}$ is the $s$ th indefinite integral of $\psi$. Therefore, by Theorem 1.2, it follows that $\left(\psi_{s}\right)_{([\lambda]+s)}$ exists finitely on a set $E_{4} \subset E_{3}$, where $\mu\left(E_{4}\right)=\mu\left(E_{3}\right)$. Let $E_{5} \subset E_{4}$ be such that $\mu\left(E_{5}\right)=\mu\left(E_{4}\right)$ and $\psi_{s}^{(s)}=\psi$ on $E_{5}$. Now by [11, II; p. 77, Theorem 4.25], there is a perfect set $P \subset E_{5}$ such that $\mu\left(E_{5} \backslash P\right)<\epsilon_{2}$ and there are functions $G$ and $H$ satisfying 
(i) $\psi_{s}=G+H$,

(ii) $G^{([\lambda]+s)}$ exists continuously, and

(iii) $H_{(r)}(x)=0$ for $x \in P, r=0,1, \ldots,[\lambda]+s$.

Let $g=G^{(s)}$. Then $g^{([\lambda])}$ exists continuously. So, $H^{(s)}=\left(\psi_{s}-G\right)^{(s)}=\psi-g$ on $E_{5}$. Let $h=H^{(s)}$ on $E_{5}$. Then $\psi=g+h$ on $E_{5}$. Since $H=0$ on $P$ and $H^{(s)}$ exists on $P, H^{(s)}(x)=0$ for $x \in P$, and so $h(x)=0$ for all $x \in P$. Since for all $x \in E_{5}, \psi, g$ satisfy (note that $\psi=f$ on $\cup_{i=1}^{n} I_{i}$ )

$$
\sum_{i=0}^{k+\ell} a_{i} \psi\left(x+b_{i} t\right)=O\left(t^{[\lambda]}\right), \quad \text { as } t \rightarrow 0
$$

and

$$
\sum_{i=0}^{n} a_{i} g\left(x+b_{i} t\right)=O\left(t^{[\lambda]}\right), \quad \text { as } t \rightarrow 0,
$$

we have for all $x \in E_{5}$

$$
\sum_{i=0}^{n} a_{i} h\left(x+b_{i} t\right)=O\left(t^{[\lambda]}\right), \quad \text { as } t \rightarrow 0 .
$$

We now show that $h_{([\lambda])}$ exists finitely a.e. on $P$. Define for each $m \in \mathbb{N}^{+}$,

$$
E_{m}^{*}=\left\{x: x \in P ;\left|\sum_{i=0}^{k+\ell} a_{i} h\left(x+b_{i} t\right)\right| \leq m|t|^{[\lambda]}, \quad \text { for } 0<|t|<\frac{1}{m}\right\} .
$$

Then $P=\cup_{m=1}^{\infty} E_{m}^{*}$. Let $m$ be fixed. Let $x_{0} \in E_{m}^{*}$ be a point of outer density of $E_{m}^{*}$. We may suppose that $x_{0}=0$. Let $\eta, 0<\eta<\frac{1}{k+\ell+2}$, be arbitrary. Choose $j, 0 \leq j \leq k+\ell$, such that $a_{j} \neq 0, b_{j} \neq 0$. By reordering the terms of $A$ we may suppose that $a_{0} \neq 0, b_{0} \neq 0$. Then by Lemma 2.1 there is a $\delta_{1}$, $0<\delta_{1}<1$, such that if $0<t<\delta_{1}$ then

$$
\mu^{*}\left(B_{i}\right)>(1-\eta) t \quad \text { and } \quad \mu^{*}(C)>(1-\eta) t \quad \text { for } i=1,2, \ldots, k+\ell,
$$

where

$$
\begin{gathered}
B_{i}=\left\{u: u \in[t, 2 t] ; t+\left(b_{i}-b_{0}\right) u \in E_{m}^{*}\right\}, \quad i=1,2, \ldots, k+\ell, \\
C=\left\{u: u \in[t, 2 t] ; t-b_{0} u \in E_{m}^{*}\right\} .
\end{gathered}
$$


Fix $t \in\left(0, \min \left(\delta_{1}, 1 / 2 m\right)\right)$. Set

$$
\begin{gathered}
S_{i}=\left\{u: u \in[t, 2 t] ; t+\left(b_{i}-b_{0}\right) u \in P\right\}, \quad i=1,2, \ldots, k+\ell, \\
D=\left\{u: u \in[t, 2 t] ;\left|\sum_{i=0}^{k+\ell} a_{i} h\left(\left(t-b_{0} u\right)+b_{i} u\right)\right| \leq m|u|^{[\lambda]}\right\} .
\end{gathered}
$$

Then the $S_{i}$ 's and $D$ are measurable for $i=1,2, \ldots, k+\ell$. and $C \subset D$, $B_{i} \subset S_{i}$, and so

$$
\mu(D)>(1-\eta) t, \quad \mu\left(S_{i}\right)>(1-\eta) t, \quad \text { for } i=1,2, \ldots, k+\ell .
$$

Now, since

$$
\mu\left([t, 2 t] \backslash\left(\left(\cap_{i=1}^{k+\ell} S_{i}\right) \cap D\right)\right)<(k+\ell+1) \eta t<t,
$$

we have $\mu\left(\left(\cap_{i=1}^{k+\ell} S_{i}\right) \cap D\right)>0$. Hence there is an $u \in\left(\cap_{i=1}^{k+\ell} S_{i}\right) \cap D$, and so $t+\left(b_{i}-b_{0}\right) u \in P$, for all $i=1,2, \ldots, k+\ell$, which gives

$$
h\left(t+\left(b_{i}-b_{0}\right) u\right)=0, \quad \text { for all } i=1,2, \ldots, k+\ell .
$$

Also, since $u \in D$,

$$
\left|\sum_{i=0}^{k+\ell} a_{i} h\left(\left(t-b_{0} u\right)+b_{i} u\right)\right| \leq m|u|^{[\lambda]}
$$

and hence

$$
\left|a_{0} h(t)\right|=\left|\sum_{i=0}^{k+\ell} a_{i} h\left(\left(t-b_{0} u\right)+b_{i} u\right)\right| \leq m|u|^{[\lambda]} \leq 2^{[\lambda]} m|t|^{[\lambda]} .
$$

This shows that

$$
h(t)=O\left(t^{[\lambda]}\right), \quad \text { as } t \rightarrow 0 .
$$

Since $x_{0}=0$ is a point of outer density of $E_{m}^{*}$, it follows that

$$
h(x+t)=O\left(t^{[\lambda]}\right), \quad \text { as } t \rightarrow 0,
$$

for almost all points $x$ in $E_{m}^{*}$, and hence this also holds for almost all points $x$ in $P$. Therefore by Lemma $2.4, h_{([\lambda])}$ exists a.e. on $P$. Thus $\psi_{([\lambda])}$ exists a.e. on $P$. Since $P \subset E_{5}$ and $\mu\left(E_{5} \backslash P\right)<\epsilon_{2}$, and since $\epsilon_{2}$ is arbitrary, $\psi_{([\lambda])}$ exists a.e. on $E_{5}$. Since $E_{5} \subset E_{2} \subset \cup_{i=1}^{n} I_{i}$ and since $f=\psi$ on $\cup_{i=1}^{n} I_{i}$, which is an open set, $f_{([\lambda])}$ exists a.e. on $E_{5}$. Since $E_{5} \subset E_{2} \subset E_{1} \subset E, \mu\left(E_{5}\right)=\mu\left(E_{2}\right)$, $\mu\left(E_{1} \backslash E_{2}\right)<\epsilon_{1}$ and $\mu\left(E_{1}\right)=\mu(E)$, and since $\epsilon_{1}$ is arbitrary, $f_{([\lambda])}$ exists a.e. on $E$. This completes the proof. 
The above theorem is not true for $\lambda=k-1$ (see [6, Theorem 3.2]). However we have Theorem 3.3

Theorem 3.3. Let $k, p \in \mathbb{N}^{+}, p \leq k-1$ and let $f$ be measurable. Let

$$
\Phi_{k}(x, u ; f ; A)=O\left(u^{p}\right), \quad \text { as } u \rightarrow 0,
$$

for each point $x$ in a set $E$. If $f_{(p), a}$ exists finitely on $E$, then $f_{(p)}$ exists a.e. on E. More generally, if

$$
-\infty<\underline{f}_{(p), a} \leq \bar{f}_{(p), a}<\infty \text { on } E,
$$

then $f_{(p-1)}$ exists finitely and

$$
-\infty<\underline{f}_{(p)} \leq \bar{f}_{(p)}<\infty \text { a.e. on } E .
$$

To prove the theorem, we need the following lemma.

Lemma 3.4. Let $k, p \in \mathbb{N}^{+}$and let $f$ be measurable. Let for all $m \in \mathbb{N}^{+}$,

$$
\begin{aligned}
& E_{m}=\left\{x: f_{(p), a}(x)\right. \text { exists finitely and } \\
& \left.\qquad\left|\Phi_{k}(x, u ; f ; A)\right|<m|u|^{p} \text { for } 0<|u|<\frac{1}{m}\right\} .
\end{aligned}
$$

Then $f_{(p)}$ exists a.e. on $E_{m}$.

Proof. Without loss of generality we may assume that $a_{0} \neq 0, b_{0} \neq 0$ in $A=\left\{a_{0}, a_{1}, \ldots, a_{k+\ell} ; b_{0}, b_{1}, \ldots, b_{k+\ell}\right\}$. Let $x_{0} \in E_{m}$ be a point of outer density of $E_{m}$. We suppose that

$$
x_{0}=0=f\left(x_{0}\right)=f_{(1), a}\left(x_{0}\right)=\ldots=f_{(p), a}\left(x_{0}\right) .
$$

Let $0<\epsilon<1$. Let

$$
G=\left\{x:|f(x)| \leq \frac{\epsilon|x|^{p}}{p !}\right\} .
$$

Then $G$ is measurable and $0 \in G$ is a point of density of $G$. Set $H=E_{m} \cap G$. Then 0 is a point of outer density of $H$. Let $0<\eta<\frac{\epsilon}{2 k+2 \ell}$. Then by Lemma 2.1, there is a $\delta>0$ such that, if $0<u<\delta$ then

$$
\mu^{*}(B)>(1-\eta) u, \quad \mu^{*}\left(C_{j}\right)>(1-\eta) u,
$$

where

$$
B=\left\{v \in[u, 2 u]: \frac{u+v}{2} \in H\right\},
$$




$$
C_{j}=\left\{v \in[u, 2 u]: \lambda_{j} u+\mu_{j} v \in H\right\}, \quad \text { for } 1 \leq j \leq k+\ell,
$$

where the $\lambda_{j}$ 's and $\mu_{j}$ 's are given as follows:

$$
\lambda_{j}=\frac{1+\frac{b_{j}}{b_{0}}}{2}, \quad \mu_{j}=\frac{1-\frac{b_{j}}{b_{0}}}{2} \quad \text { for } \quad 1 \leq j \leq k+\ell .
$$

Fix $u \in\left(0, \min \left(\delta, \frac{\left|b_{0}\right|}{m}\right)\right)$. Let

$$
S=\left\{v \in[u, 2 u]:\left|\Phi_{k}\left(\frac{u+v}{2}, \frac{u-v}{2 b_{0}}\right)\right|<m\left|\frac{u-v}{2 b_{0}}\right|^{p}\right\}
$$

and

$$
T_{j}=\left\{v \in[u, 2 u]:\left|f\left(\lambda_{j} u+\mu_{j} v\right)\right| \leq \frac{\epsilon\left|\lambda_{j} u+\mu_{j} v\right|^{p}}{p !}\right\}, \quad \text { for } 1 \leq j \leq k+\ell .
$$

Since $f$ is measurable, $S$ and $T_{j}$ are measurable. Also $B \subset S, C_{j} \subset T_{j}$, and hence

$$
\mu(S)>(1-\eta) u, \quad \mu\left(T_{j}\right)>(1-\eta) u .
$$

Therefore

$$
\mu\left(\cap_{j}\left(S \cap T_{j}\right)\right)>(1-2(k+\ell) \eta) u>(1-\epsilon) u .
$$

Hence

$$
\left(\cap_{j}\left(S \cap T_{j}\right)\right) \cap(u, u+\epsilon u) \neq \emptyset .
$$

Choose $v \in\left(\cap_{j}\left(S \cap T_{j}\right)\right) \cap(u, u+\epsilon u)$. Then

$$
0<v-u<\epsilon u<u,
$$

and so

$$
\left|\Phi_{k}\left(\frac{u+v}{2}, \frac{u-v}{2 b_{0}}\right)\right|<m\left|\frac{u-v}{2 b_{0}}\right|^{p}<m\left|\frac{\epsilon u}{2 b_{0}}\right|^{p},
$$

which gives

$$
\left|\sum_{i=0}^{k+\ell} a_{i} f\left(\frac{u+v}{2}+b_{i} \frac{u-v}{2 b_{0}}\right)\right|<m\left|\frac{\epsilon u}{2 b_{0}}\right|^{p} .
$$

Hence

$$
\left|a_{0}\right| \cdot|f(u)|<m\left|\frac{\epsilon u}{2 b_{0}}\right|^{p}+\sum_{i=1}^{k+\ell}\left|a_{i}\right| \cdot\left|f\left(\frac{\left(1+\frac{b_{i}}{b_{0}}\right) u}{2}+\frac{\left(1-\frac{b_{i}}{b_{0}}\right) v}{2}\right)\right| .
$$


Since $v \in T_{i}$ for $1 \leq i \leq k+\ell$,

$$
\begin{aligned}
\left|a_{0}\right| \cdot|f(u)| & <m\left|\frac{\epsilon u}{2 b_{0}}\right|^{p}+\sum_{i=1}^{k+\ell} \frac{\epsilon\left|a_{i}\right| \cdot\left|\lambda_{i} u+\mu_{i} v\right|^{p}}{p !} \\
& \leq m\left|\frac{\epsilon u}{2 b_{0}}\right|^{p}+\frac{\epsilon}{p !} \sum_{i=1}^{k+\ell}\left|a_{i}\right|\left(\left|\lambda_{i}\right|+2\left|\mu_{i}\right|\right)^{p} u^{p} \\
& \leq \epsilon\left[\frac{m}{\left|2 b_{0}\right|^{p}}+\frac{1}{p !} \sum_{i=1}^{k+\ell}\left|a_{i}\right|\left(\left|\lambda_{i}\right|+2\left|\mu_{i}\right|\right)^{p}\right] u^{p} .
\end{aligned}
$$

This shows that $\frac{f(u)}{u^{p}} \rightarrow 0$ as $u \rightarrow 0^{+}$.

Similarly, it can be shown that $\frac{f(u)}{u^{p}} \rightarrow 0$ as $u \rightarrow 0^{-}$. This completes the proof of the lemma.

Proof of the theorem. The sequence $\left\{E_{m}\right\}$, defined in Lemma 3.4, is nondecreasing and $E \subset \cup_{m=1}^{\infty} E_{m}$. By Lemma 3.4, $f_{(p)}$ exists a.e. on $E_{m}$, and so the first part follows.

For the last part we proceed exactly as in Lemma 3.4 and in the first part of the theorem, but with the following changes:

$$
\begin{aligned}
& E_{m}=\left\{x:-m<\underline{f}_{(p), a}(x) \leq \bar{f}_{(p), a}(x)<m\right. \text { and } \\
&\left.\left|\Phi_{k}(x, u ; f ; A)\right|<m|u|^{p} \text { for } 0<|u|<\frac{1}{m}\right\},
\end{aligned}
$$

with the assumption that

$$
x_{0}=0=f\left(x_{0}\right)=f_{(1), a}\left(x_{0}\right)=\ldots=f_{(p-1), a}\left(x_{0}\right),
$$

and

$$
\begin{gathered}
G_{m}=\left\{x:|f(x)| \leq \frac{m|x|^{p}}{p !}\right\}, \\
T_{j}=\left\{v \in[u, 2 u]:\left|f\left(\lambda_{j} u+\mu_{j} v\right)\right| \leq \frac{m\left|\lambda_{j} u+\mu_{j} v\right|^{p}}{p !}\right\}, \text { for } 1 \leq j \leq k+\ell,
\end{gathered}
$$

other sets in Lemma 3.4 remaining unchanged. Proceeding as in Lemma 3.4,

$$
\left|a_{0}\right| \cdot|f(u)| \leq\left[\frac{m \epsilon^{p}}{\left|2 b_{0}\right|^{p}}+\frac{m}{p !} \sum_{i=1}^{k+\ell}\left|a_{i}\right|\left(\left|\lambda_{i}\right|+2\left|\mu_{i}\right|\right)^{p}\right] u^{p}
$$

showing that $f(u)=O\left(u^{p}\right)$ as $u \rightarrow 0^{+}$, and similarly $f(u)=O\left(u^{p}\right)$ as $u \rightarrow 0^{-}$, and the rest is clear. 
Corollary 3.5. Under the hypotheses of Theorem 3.3, if

$$
-\infty<\underline{f}_{(p), a} \leq \bar{f}_{(p), a}<\infty \quad \text { on } E
$$

then $f_{(p)}$ exists finitely a.e. on E.

The proof follows from Theorem 3.3 and Theorem 2.2 of [6].

\section{References}

[1] J. M. Ash, Generalizations of the Riemann derivative, Trans. Amer. Math. Soc. 126 (1967), 181-199.

[2] J. A. Bergin, A new characterization of Cesaro-Perron integrals using Peano derivatives, Trans. Amer. Math. Soc. 228 (1977), 287-305.

[3] H. Fejzic and C. E. Weil, Repairing the proof of a classical differentiation result, Real Analysis Exchange 19 (1993/4), no. 2, 639-643.

[4] P. R. Halmos, Measure theory, D. Van Nostrand Co., 1950.

[5] J. Marcinkiewicz and A. Zygmund, On the differentiability of functions and summability of trigonometric series, Fund. Math. 26 (1936), 1-43.

[6] S. N. Mukhopadhyay and S. Mitra, An extension of a theorem of Marcinkiewicz and Zygmund on differentiability, Fund. Math. 151 (1996), 21-38.

[7] S. N. Mukhopadhyay and S. Mitra, Measurability of Peano derivates and approximate Peano derivates, Real Analysis Exchange 20 (1994/5), no. $2,768-775$.

[8] L. A. Rubel, A pathological Lebesgue measurable function, J. London Math. Soc. 38 (1963), 1-4.

[9] W. Sierpinski, Sur la question de la measurabilité de la base de M. Hamel, Fund. Math. 1 (1920), 105-111.

[10] B. S. Thomson, Symmetric properties of real functions, Marcel Dekker, Inc., 1994.

[11] A. Zygmund, Trigonometric series, Cambridge University Press, 1968. 
S. N. Mukhopadhyay and S. Mitra 